ARTICLE

Check for updates

https://doi.org/10.1038/s41467-020-18380-1

\title{
Intermediate-phase-assisted low-temperature formation of $\gamma$-CsPbl 3 films for high-efficiency deep-red light-emitting devices
}

Chang $\mathrm{Yi}^{1}{ }^{1,4}$, Chao Liu ${ }^{1} 4$, Kaichuan Wen ${ }^{1}$, Xiao-Ke Liu (D) ${ }^{2}$, Hao Zhang ${ }^{1}$, Yong $\mathrm{Yu}^{2}$, Ning Fan ${ }^{1}$, Fuxiang $\mathrm{Ji}^{2}$,

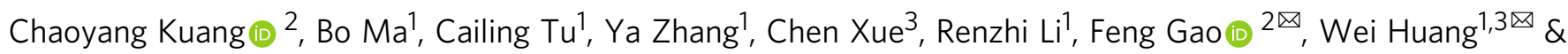
Jianpu Wang (10) ${ }^{1 凶}$

Black phase $\mathrm{CsPbl}_{3}$ is attractive for optoelectronic devices, while usually it has a high formation energy and requires an annealing temperature of above $300^{\circ} \mathrm{C}$. The formation energy can be significantly reduced by adding $\mathrm{HI}$ in the precursor. However, the resulting films are not suitable for light-emitting applications due to the high trap densities and low photoluminescence quantum efficiencies, and the low temperature formation mechanism is not well understood yet. Here, we demonstrate a general approach for deposition of $\boldsymbol{\gamma}-\mathrm{CsPb}_{3}$ films at $100^{\circ} \mathrm{C}$ with high photoluminescence quantum efficiencies by adding organic ammonium cations, and the resulting light-emitting diode exhibits an external quantum efficiency of $10.4 \%$ with suppressed efficiency roll-off. We reveal that the low-temperature crystallization process is due to the formation of low-dimensional intermediate states, and followed by interionic exchange. This work provides perspectives to tune phase transition pathway at low temperature for $\mathrm{CsPbl}_{3}$ device applications.

\footnotetext{
${ }^{1}$ Key Laboratory of Flexible Electronics (KLOFE) \& Institute of Advanced Materials (IAM), Nanjing Tech University (NanjingTech), 30 South Puzhu Road, Nanjing 211816, China. ${ }^{2}$ Department of Physics, Chemistry, and Biology (IFM), Linköping University, SE-58183 Linköping, Sweden. ${ }^{3}$ Frontiers Science Center for Flexible Electronics (FSCFE), Shaanxi Institute of Flexible Electronics (SIFE), Xi'an Institute of Biomedical Materials \& Engineering (IBME), Northwestern Polytechnical University (NPU), 127 West Youyi Road, Xi'an 710072, China. ${ }^{4}$ These authors contributed equally: Chang Yi, Chao Liu. ${ }^{凶}$ email: feng.gao@liu.se; iamwhuang@nwpu.edu.cn; iamjpwang@njtech.edu.cn
} 
$\Lambda$ ll-inorganic halide perovskites such as $\mathrm{CsPbX}_{3}(\mathrm{X}=\mathrm{Cl}$, $\mathrm{Br}, \mathrm{I})$ are attractive materials for light emitters and photovoltaic applications due to their potential in overcoming long-term stability issues of organic-inorganic hybrid halide perovskites ${ }^{1-5}$. The low-temperature solution-processed phasestable $\mathrm{CsPbX}_{3}$ perovskites are mainly based on bromide and chloride with suitable tolerance factors ${ }^{6}$. Optically active $\mathrm{CsPbI}_{3}$ black phases ( $\alpha$ (cubic), $\beta$ (tetragonal) or $\gamma$ (orthorhombic)) usually require high annealing temperature $\left(300-370^{\circ} \mathrm{C}\right)$ to overcome the energy barrier for phase transition ${ }^{7,8}$. Moreover, the $\mathrm{CsPb}_{3}$ black phases can readily transform to yellow-phase $\delta$ $\mathrm{CsPb}_{3}$ in ambient conditions due to the thermodynamic instability ${ }^{9-11}$, inhibiting their application in optoelectronic devices ${ }^{12,13}$. In perovskite solar cells, a general method of forming black phases $\mathrm{CsPbI}_{3}$ at low temperature is by adding hydroiodic acid (HI) in $\mathrm{CsPbI}_{3}$ precursor solution prior to spin coating ${ }^{13,14}$. It has been observed that the judicious amount of $\mathrm{HI}$ would decompose the solvent dimethylformamide (DMF) to form dimethylammonium iodide ${ }^{15}$, while the mechanism of how this process affects the crystallization of $\mathrm{CsPb}_{3}$ is still under intensive debate with two arguments. One argument is that the formed DMA would sublimate and lead to a fast crystallization of $\mathrm{CsPb}_{3}{ }_{3}^{4,16,17}$. Another argument is that the DMA becomes the part of the crystal structure and the formed black phase is not ${ }_{\mathrm{Cs}} \mathrm{PbI}_{3}$ but $\mathrm{Cs}_{x} \mathrm{DMA}_{1-x} \mathrm{PbI}_{3}{ }^{18,19}$.

For $\mathrm{CsPbI}_{3}$-based light-emitting diodes (LEDs) applications, the low-temperature HI doping method is difficult to achieve high performance devices, mainly due to the high trap density and strong nonradiative recombination with those perovskite films (typical photoluminescence quantum efficiency (PLQE) < $1 \%)^{20}$. Alternatively, high-efficiency LEDs has been demonstrated based on $\mathrm{CsPbI}_{3}$ quantum dots (QDs) ${ }^{21,22}$. However, those colloidal QDs are synthesized ex situ in flasks by the hot-injection method, which usually requires a temperature above $170^{\circ} \mathrm{C}$ and complicated processing condition ${ }^{21-24}$. In addition, usually those perovskite QD-based LEDs only show high efficiency at low current densities with strong efficiency roll-off due to the strong nonradiative Auger process in perovskite QDs ${ }^{21,25,26}$. In this work, we report an effective approach for achieving high quality $\gamma-\mathrm{CsPbI}_{3}$ at low annealing temperature $\left(\sim 100^{\circ} \mathrm{C}\right)$ for high performance LEDs applications. More importantly, we reveal that the low-temperature formation process of black phase $\mathrm{CsPb}_{3} \mathrm{can}$ be generally observed when intermediate states are formed, followed by an interionic exchange in the presence of large organic ammonium cations.

\section{Results}

Low temperature formed $\gamma$-CsPbI ${ }_{3}$ films. A DMF precursor solution of imidazolium iodide (IZI), CsI, and $\mathrm{PbI}_{2}$ with a molar ratio of 4:1.5:1 (referred as IZI-CsPbI ${ }_{3}$ ) is spin coated onto polyethylenimine ethoxylated (PEIE) modified $\mathrm{ZnO}$ substrates (referred as $\mathrm{ZnO} / \mathrm{PEIE}$ ). We note that $\mathrm{ZnO} / \mathrm{PEIE}$ has been widely used as an electron transporting layer in perovskite $\mathrm{LEDs}^{27}$. After thermal annealing at $100{ }^{\circ} \mathrm{C}$ for $5 \mathrm{~min}$, the IZI-CsPbI ${ }_{3}$ film shows X-ray diffraction (XRD) peak of 14.3 and $28.9^{\circ}$ without any splitting (Fig. 1a), corresponding to the (110) and (220) crystal planes of $\gamma-\mathrm{CsPbI}_{3}$, respectively. An absorbance edge at $\sim 1.75 \mathrm{eV}$ and a photoluminescence (PL) peak at $\sim 700 \mathrm{~nm}$ are also observed for this film (Fig. 1b). These results are consistent with the characteristics of $\gamma-\mathrm{CsPb}_{3}$ obtained through thermal annealing above the transition temperature (around $310^{\circ} \mathrm{C}$ ) and rapid cooling process ${ }^{8,13}$. In addition, the scanning electron microscopic (SEM) measurement shows that the IZI-CsPbI film is discrete, consisting of particles with an average size of $\sim 80 \mathrm{~nm}$ (Fig. 1c). The film shows good emission properties with PLQE reaching up to $38 \%$ (Fig. 1d). Time-correlated single photon counting measurement shows that the PL lifetime increases with the increasing amount of IZI (Supplementary Fig. 1a), suggesting that the nonradiative recombination of the $\gamma-\mathrm{CsPb}_{3}$ is suppressed with increasing IZI. This result is consistent with the PLQE result (Supplementary Fig. 1b). More importantly, the IZI$\mathrm{CsPbI}_{3}$ film exhibits negligible degradation after exposing for 36 days in ambient air at room temperature with $80 \%$ relative humidity (Fig. 1d). In contrast, the regular $\gamma-\mathrm{CsPb}_{3}$ obtained from the high-temperature annealing process can only retain for $4 \mathrm{~h}$ in the same environment (Supplementary Fig. 2). The PL intensity of IZI-CsPbI film dropped to $50 \%$ over 8 days in the ambient air (Supplementary Fig. 3), suggesting significantly improved phase and optical stability compared to previously reported results ${ }^{4,19}$.

The mechanism behind low temperature formed $\gamma-\mathrm{CsPbI}_{3}$ films. To investigate the mechanism of IZI on facilitating the formation of $\gamma$-CsPbI ${ }_{3}$ perovskite at the low temperature, we monitor the crystal phase evolution of the as-spun IZI-CsPbI film by XRD measurements under various annealing time (Fig. 2a). At early stage of the thermal annealing process (10-15 s), CsI with the peak at $27.6^{\circ}$ remains unchanged, while an intermediate phase with peaks at 11.3 and $25.4^{\circ}$ is formed. This intermediate phase can be assigned to one dimensional (1D) $\mathrm{IZPbI}_{3}$, since their XRD peaks are consistent (Fig. 2a red line and Supplementary Table 1). Upon further annealing ( $\sim 30-60 \mathrm{~s})$, both the XRD peaks $\left(11.3,25.4\right.$, and $\left.27.6^{\circ}\right)$ of intermediate phase $\mathrm{IZPbI}_{3}$ and CsI disappear. In the meantime, XRD peaks (14.3 and $28.9^{\circ}$ ) of $\gamma-\mathrm{CsPbI}_{3}$ perovskite appear. These facts suggest that the 1D $\mathrm{IZPbI}_{3}$ perovskite transforms to the $\gamma-\mathrm{CsPbI}$ perovskite during the low-temperature annealing process. This transformation process requires an interionic exchange process of $\mathrm{IZ}^{+}$ embedded in face-shared $\mathrm{PbI}_{6}$ chains with external $\mathrm{Cs}^{+}$. The corresponding morphology of IZI-CsPbI films annealed at $100^{\circ} \mathrm{C}$ for various time durations is also monitored by SEM measurement (Supplementary Fig. 4). The unannealed film displays a dense, planar morphology (Supplementary Fig. 4a). With a short-time annealing $(t=10 \mathrm{~s})$, mounts of small grains of about $40 \mathrm{~nm}$ emerge (Supplementary Fig. $4 \mathrm{~b}$ ), corresponding to the intermediate phase. By extending the annealing time duration to $15 \mathrm{~s}$, the small grains grow bigger and the layer becomes discrete (Supplementary Fig. 4c), corresponding to the mixed phase with $1 \mathrm{D}$ and 3D. When annealed over $30 \mathrm{~s}$, the discrete $\gamma-\mathrm{CsPb}_{3}$ grains with an average size of $\sim 80 \mathrm{~nm}$ form and disperse on the $\mathrm{ZnO} /$ PEIE substrate (Supplementary Fig. 4d-f).

We find that without the underneath ultrathin PEIE layer, the low-temperature phase transformation can be still observed by XRD measurement (Supplementary Fig. 5). It cannot be formed at $100^{\circ} \mathrm{C}$ without $\mathrm{ZnO}$ layer, where both the $1 \mathrm{D}$ phase and CsI remain unchanged even after 10 min annealing (Supplementary Fig. 6). UV-vis absorption spectra measurement result is consistent with the above XRD result (Supplementary Fig. 7). These results suggest that during the formation of $\gamma-\mathrm{CsPbI}_{3}$ from the intermediate phase $\mathrm{IZPb}_{3}$, the $\mathrm{ZnO}$ substrate plays important roles in the interionic exchange process.

We then use X-ray photoelectron spectroscopy (XPS) measurement to reveal the role of the $\mathrm{ZnO}$ substrate in the interionic exchange process. Particularly, the chemical interaction between the films (IZI, IZI-CsPbI ${ }_{3}$ ) and $\mathrm{ZnO}$ is investigated. Figure $2 \mathrm{~b}$ shows the high resolution XPS spectra of $\mathrm{N} 1 s, \mathrm{O} 1 s$, and Zn $2 p$ of these films. The XPS spectrum of IZI prepared on ITO substrate shows two $\mathrm{N} 1 s$ peaks at around $401.7 \mathrm{eV}(\mathrm{N}-3)$ and $400.2 \mathrm{eV}(\mathrm{N}-$ 1), respectively (Fig. 2b black and pink line). When IZI film is on top of $\mathrm{ZnO}$ layer, the N-3 peak disappears and a new peak 
a

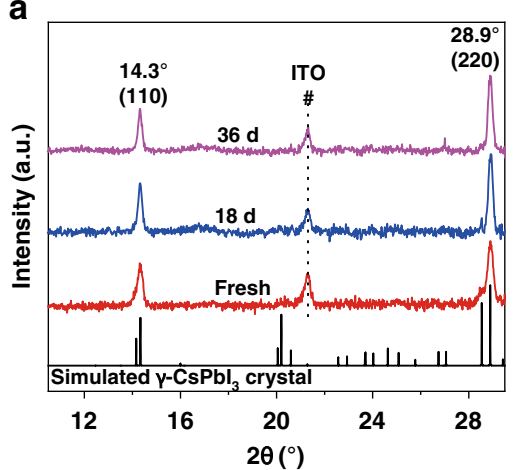

C

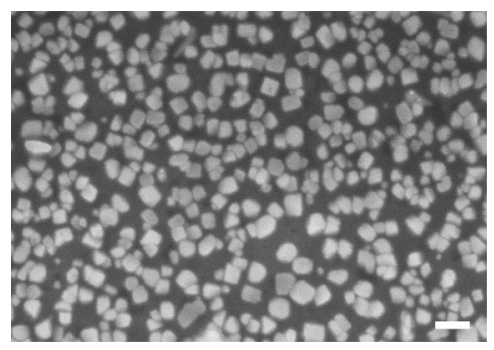

b

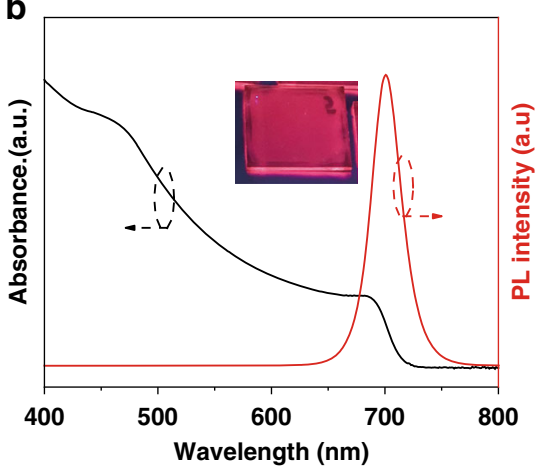

d

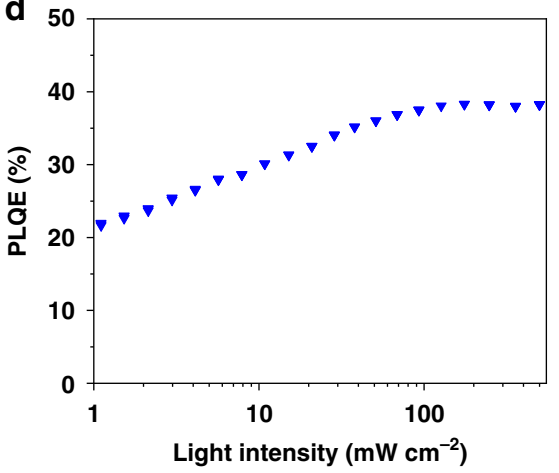

Fig. 1 Characteristics of IZI-CsPbl $\mathbf{3}$ film on ZnO/PEIE substrate. a XRD pattern after exposed in the air with $80 \%$ relative humidity for various durations. Black line is the simulated powder patterns of $\gamma-\mathrm{CsPbl}_{3}{ }^{8}$. b UV-vis and PL spectrum. Inset shows the image under UV light. c SEM image (scale bar, 200 $\mathrm{nm}$ ), and d Excitation-intensity-dependent PLQE.

positioning at a lower binding energy of $398.8 \mathrm{eV}$ shows up. The two peaks of the $\mathrm{ZnO} / \mathrm{IZI}$ film are in well agreement with those of imidazole (IZ), and the peak at $398.8 \mathrm{eV}$ can be attributed to the $\mathrm{N}-2$ of IZ (Fig. 2b blue line) ${ }^{28,29}$. The above XPS result indicates that the $\mathrm{ZnO}$ can deprotonate the $\mathrm{IZ}^{+}$cation of IZI. Importantly, this deprotonation process can be also observed in the IZI-CsPbI film with $\mathrm{ZnO}$ and $\mathrm{ZnO} / \mathrm{PEIE}$ substrate, respectively (Fig. $2 \mathrm{~b}$ and Supplementary Fig. 8). Moreover, compared to the bare $\mathrm{ZnO}$ film on ITO, the $\mathrm{ZnO} / \mathrm{IZI}$ and $\mathrm{ZnO} / \mathrm{IZI}-\mathrm{CsPbI}_{3}$ films show significantly suppressed XPS peak at $530 \mathrm{eV}$ ascribed to O $1 s$ of $\mathrm{Zn}-\mathrm{O}$ bonding (Fig. $2 c$ black line) ${ }^{30,31}$, and their peaks of the $\mathrm{Zn} 2 p$ of $\mathrm{Zn}-\mathrm{O}$ bonding shift to higher energies (Fig. 2d). These further XPS observations can be explained by the presence of $\mathrm{H}^{+}$in the $\mathrm{Zn}-\mathrm{O}-\mathrm{H}$ bonding which decreases electron cloud density around $\mathrm{Zn}$ atoms ${ }^{32}$, consistent with the result of deprotonation of the $\mathrm{IZ}^{+}$ cation by $\mathrm{ZnO}$. Figure 2e shows a scheme of this deprotonation process of $\mathrm{IZ}^{+}$by $\mathrm{ZnO}$. In addition, attenuated total reflection Fourier-transform infrared (ATR-FTIR) spectroscopy measurement result can further confirm this scenario. As shown in Supplementary Fig. 9, the N-H stretching vibration at $3260 \mathrm{~cm}^{-1}$ of IZI in the films on the $\mathrm{ZnO} / \mathrm{PEIE}$ substrate is shifted to higher wavenumber $\left(3330 \mathrm{~cm}^{-1}\right)$. Correspondingly, the broad stretching vibration of $\mathrm{Zn}-\mathrm{O}$ is red shifted from 556 to $538 \mathrm{~cm}^{-1,33}$. And the signature peak of IZ at about $3130(\mathrm{CH}$ stretching), $1543(\mathrm{NH}$ bend), 1328 ( $\mathrm{CH}$ bend), 1263 (ring breathing), 1055 ( $\mathrm{CH}$ bend), 841 (ring bend), 757 ( $\mathrm{CH}$ out-of-plane bend), and 658 (torsion) $\mathrm{cm}^{-1}$ are clearly observed ${ }^{34}$. We note that the similar deprotonation process can also be observed in films with the intermediate phase of $\mathrm{IZPbI}_{3}$ (Supplementary Fig. 10) and $\mathrm{FAPbI}_{3}$ perovskites ${ }^{35}$.

On the basis of the above phase evolution and chemical elementary analysis, we can have a clear picture of the mechanism of the $\gamma-\mathrm{CsPb}_{3}$ formation process at low temperature, as shown in Fig. 2f. It first forms an intermediate phase $\mathrm{IZPbI}_{3}$, followed by the formation of $\gamma-\mathrm{CsPb}_{3}$ through the interionic exchange of $\mathrm{IZ}^{+}$ with external $\mathrm{Cs}^{+}$in the process of the deprotonation of $\mathrm{IZ}^{+}$with $\mathrm{ZnO}$. The overall chemical reaction of phase formation can be presented as follows:

Stage 1: $\mathrm{PbI}_{2}+\mathrm{C}_{3} \mathrm{~N}_{2} \mathrm{H}_{5} \mathrm{I}+\mathrm{Cs}^{+} \rightarrow\left(\mathrm{C}_{3} \mathrm{~N}_{2} \mathrm{H}_{5}\right) \mathrm{PbI}_{3}+\mathrm{Cs}^{+}$ forming intermediate phase,

Stage $2: 2\left(\mathrm{C}_{3} \mathrm{~N}_{2} \mathrm{H}_{5}\right) \mathrm{PbI}_{3}+2 \mathrm{Cs}^{+}+2 \mathrm{ZnO} \rightarrow 2 \mathrm{CsPbI}_{3}+2 \mathrm{C}_{3}$ $\mathrm{N}_{2} \mathrm{H}_{4}+\mathrm{Zn}(\mathrm{OH})_{2}+\mathrm{Zn}^{2+}$ ion exchange and forming $\gamma-\mathrm{CsPb}_{3}$.

Since the $\mathrm{ZnO}$ induced deprotonation process likely mainly occurs at the $\mathrm{ZnO}$ interface, it is interesting to investigate how thick the perovskite can be formed by this approach. Supplementary Fig. 11 shows the absorbance of the films with various thickness fabricated from different concentration of precursor solutions. It shows the absorbance at $687 \mathrm{~nm}$ from the black phase $\mathrm{CsPbI}_{3}$ increases linearly with the film thickness $<200 \mathrm{~nm}$. Above $200 \mathrm{~nm}$, the absorbance saturates and declines. This result suggests that the $\mathrm{ZnO}$ substrate can facilitate the $\gamma-\mathrm{CsPbI}_{3}$ formation within the film thickness of $200 \mathrm{~nm}$.

Kinetic of phase transition. We further investigate the crystallization kinetics of the $\gamma-\mathrm{CsPI}_{3}$ films with various IZI contents by using time-dependent UV-vis spectroscopy. The reaction progress of these films with different contents of IZI in precursor solutions (IZI/PbI 2 molar ratio is $x, x=0,0.5,1,2,3,4$, and 5) were monitored through change in absorbance $(A(t))$ at $\sim 687 \mathrm{~nm}$ (Fig. 1b). The value of formed $\gamma$-phase fraction $\chi(t)$ is defined as:

$$
\chi(t)=\frac{A(t)}{A\left(t_{\mathrm{end}}\right)},
$$



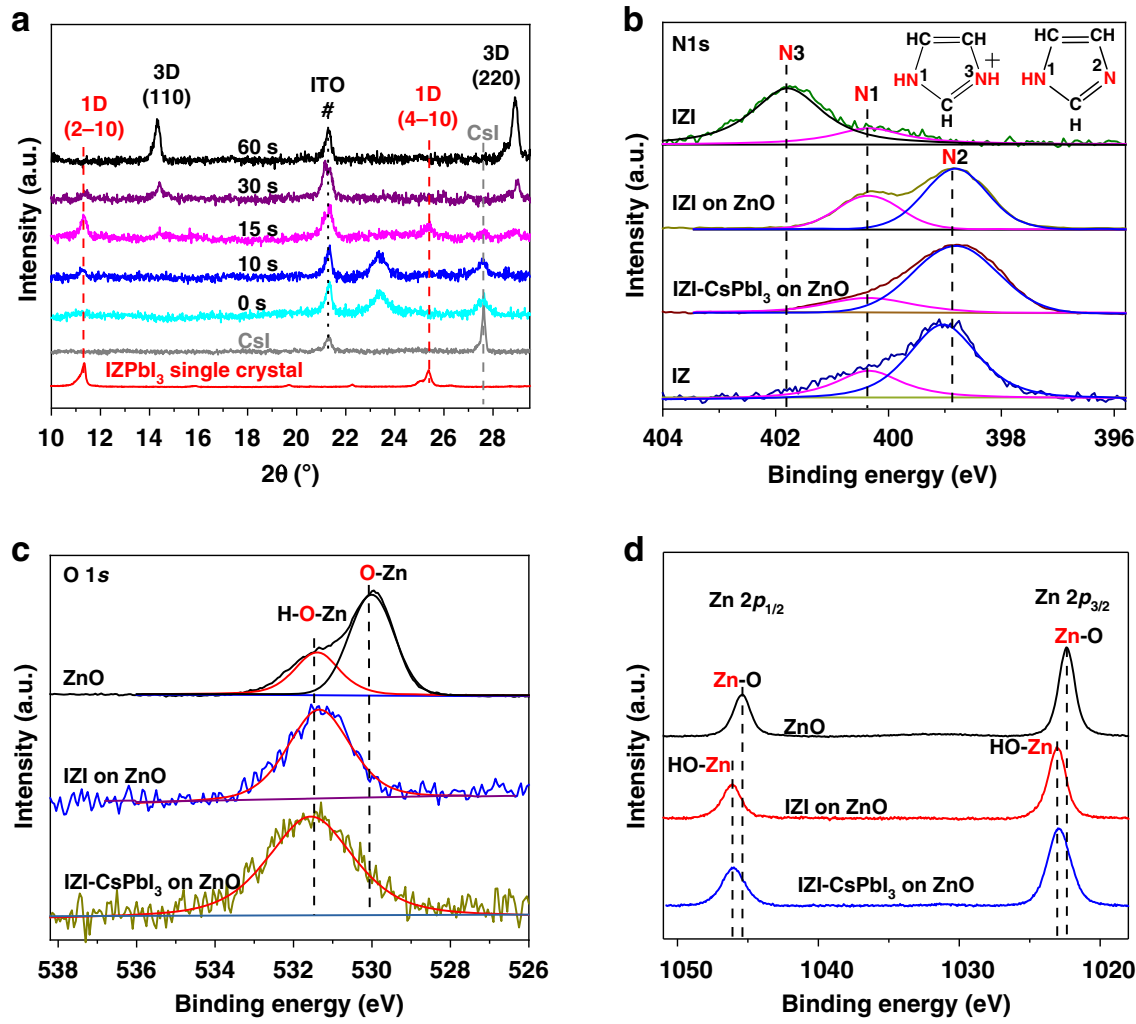

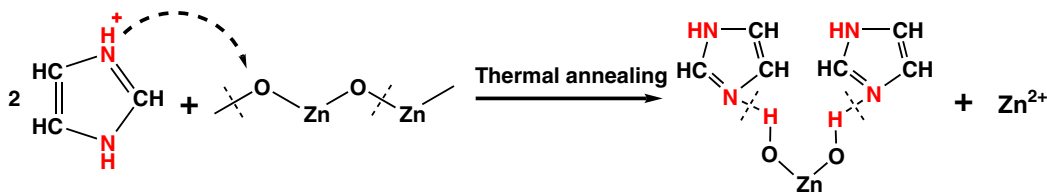

$\mathbf{f}$ $\mathrm{Pbl}_{2}$

$\mathrm{IZPb}_{3}$ $\gamma-\mathrm{CsPbl}_{3}$

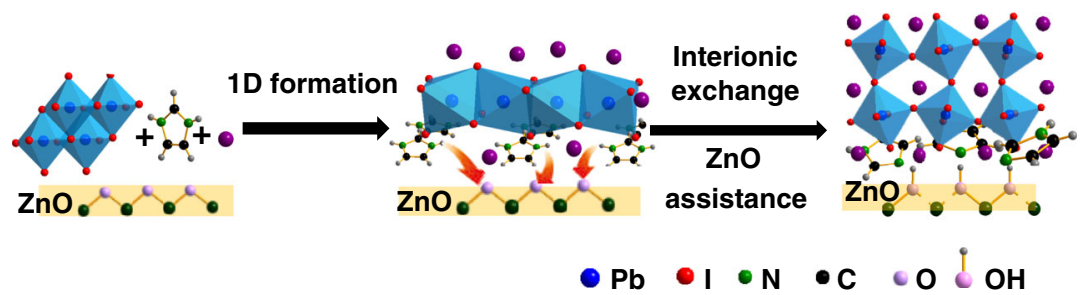

Fig. 2 Formation process of $\boldsymbol{\gamma}$ - $\mathbf{C s P b l}_{\mathbf{3}}$ perovskite on $\mathbf{Z n O} / \mathrm{PEIE}$ substrate. a XRD patterns of IZI-CsPbl ${ }_{3}$ film on $\mathrm{ZnO} / \mathrm{PEIE}$ substrate with different annealing time at $100{ }^{\circ} \mathrm{C}$. XPS spectra of (b) $\mathrm{N} 1 s,(\mathbf{c}) \mathrm{O} 1 \mathrm{~s}$, (d) $\mathrm{Zn} 2 p_{1 / 2}$ and $\mathrm{Zn} 2 p_{3 / 2}$ for IZI, IZI-CsPbl film on ZnO substrate and for IZI, IZ film on ITO substrate, respectively. e Schematic diagram of deprotonation of $\mathrm{IZ}^{+}$with $\mathrm{ZnO}$. $\mathbf{f}$ Mechanism of the $\gamma-\mathrm{CsPb}_{3}$ formation process at low temperature on $\mathrm{ZnO}$ substrate.

where the $A\left(t_{\text {end }}\right)$ represents the maximum absorbance at the final state. As shown in Fig. 3a, the rate of $\gamma$-phase formation increases with the increasing amount of IZI at a constant annealing temperature of $90^{\circ} \mathrm{C}$. And Fig. $3 \mathrm{~b}$ shows that the rate also increases with increasing annealing temperature when the IZI content is constant. More detailed measurement results on different mole ratios and various annealing temperature are shown in Supplementary Fig. 12. The activation energy of the $\gamma-\mathrm{CsPI}_{3}$ formation for various IZI contents can be estimated by using the Mittemeijer model ${ }^{36}$, as shown in Supplementary Fig. 12g. Figure 3c shows a summary of the estimated activation energies barrier, which can be significantly decreased from 150 to $29 \mathrm{~kJ} \mathrm{~mol}^{-1}$ with increasing molar ratio of IZI to $\mathrm{PbI}_{2}$ from 0.5 to 5 .
Generality of the low temperature formed $\gamma-\mathrm{CsPbI}_{3}$ films. In order to further demonstrate the generality of the low-temperature formation of black $\mathrm{CsPbI}_{3}$ through the intermediates, we add various $\mathrm{RNH}_{3}{ }^{+}$-based large organic cations, such as butylammonium iodine (BAI), hexylammonium iodine (HAI), phenethylammonium iodine (PEAI), and naphthylethylammonium iodine (NMAI) into the $\mathrm{CsPbI}_{3}$ precursor and spin coated on top of $\mathrm{ZnO}$ substrates. As shown in Supplementary Fig. 13, all those films exhibit formation of intermediates, decomposition, and interionic exchange during the annealing process. And finally, the black $\mathrm{CsPb}_{3}$ films form at $100^{\circ} \mathrm{C}$. Therefore, we believe that the process we plotted in Fig. $2 \mathrm{f}$ is general for forming black $\mathrm{CsPb}_{3}$ at low temperature assisted by $\mathrm{RNH}_{3}{ }^{+}$-based large organic cations. 

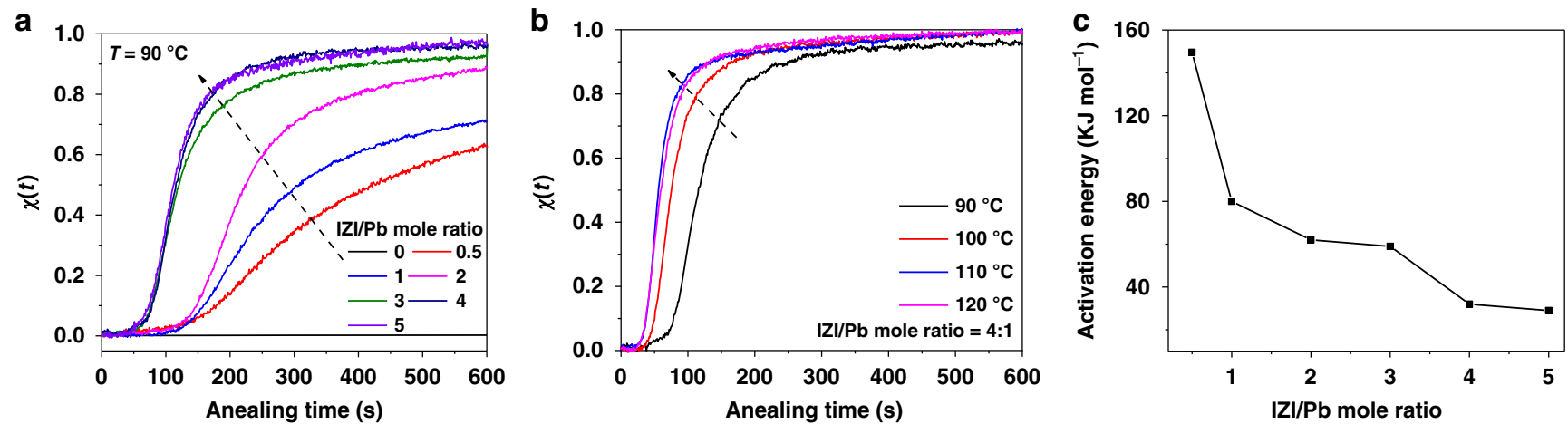

Fig. 3 Kinetic data for isothermally annealed IZI-CsPb| $\mathbf{3}$ films on ZnO/PEIE substrate. Influence of annealing time on the $\boldsymbol{\gamma}$-phase transition fraction $\chi(t)$ for (a) the IZI-CsPb| films with different mole ratio (0:1, 0.5:1, 1:1, 2:1, 3:1, 4:1, 5:1) at $90{ }^{\circ} \mathrm{C}$, and for (b) IZI-CsPb| ${ }_{3}$ with mole ratio of 4:1 at different temperature. $\chi(t)$ is defined as $A(t) / A\left(t_{\text {end }}\right), A(t)$ represents the time-dependent absorbance at $\sim 687 \mathrm{~nm}, A\left(t_{\text {end }}\right)$ represents the max absorbance. $\mathbf{c}$ The dependence of activation energies of the $\gamma$ - $\mathrm{CsPb}_{3}$ films on the IZI content.

a

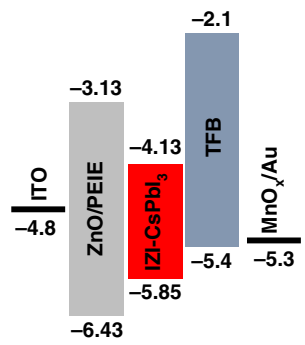

C

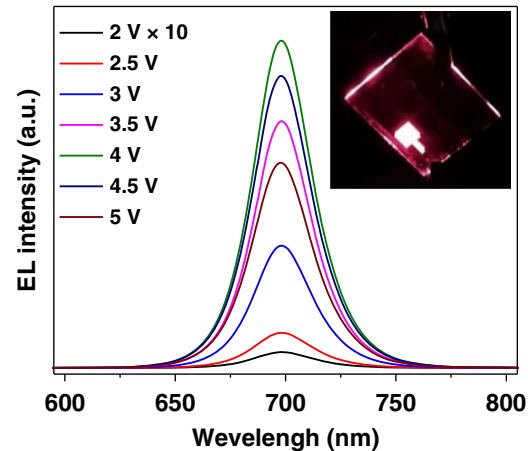

e

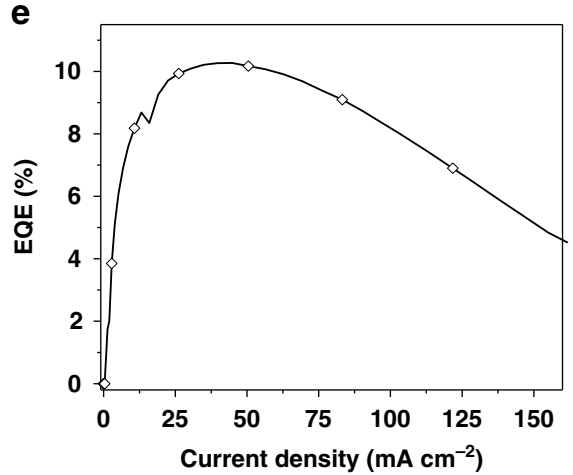

b

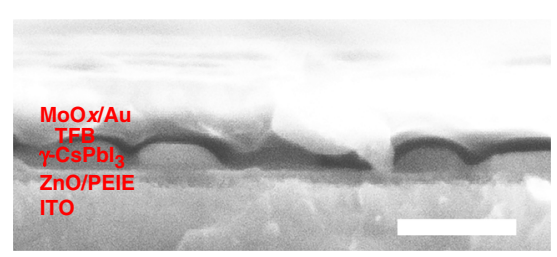

d

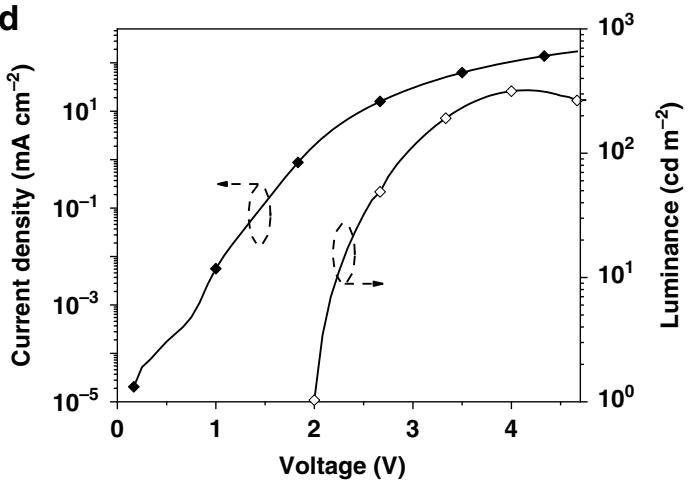

f

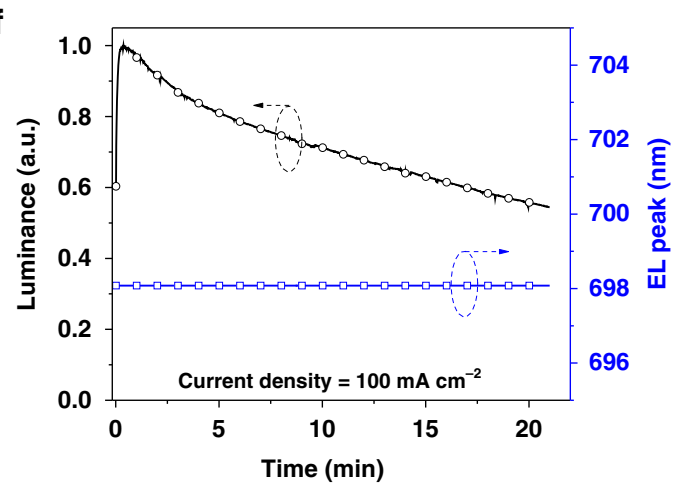

Fig. 4 IZI-CsPbl $\mathbf{3}_{\mathbf{3}}$ LED structure and optoelectronic characteristics. a Flat-band energy level diagram and b Cross-sectional SEM image (scale bar, 200 $\mathrm{nm})$. c EL spectra under various voltages. Inset shows the photograph of the deep-red device. $\mathbf{d}$ Current density and luminance versus voltage ( $J-L-V)$. e EQE versus current density $(E Q E-J)$ curves. $\mathbf{f}$ Lifetime and the maximum EL peak position measurement at $100 \mathrm{~mA} \mathrm{~cm}^{-2}$. The maximum EQE as the initial value to calculate the $T_{50}$. 
LED device performance. As shown in Fig. 4a, the LED devices have a structure of ITO/ZnO/PEIE/IZI-CsPbI $/$ /poly (9,9-dioctylfluorene-co-N-(4-butylphenyl)diphenylamine) (TFB)/molybdenum oxide $\left(\mathrm{MoO}_{x}\right) /$ gold $(\mathrm{Au})$. Detailed fabrication process can be found in "Methods." The valence bands of IZI-CsPbI ${ }_{3}$ film is obtained via ultraviolet photoelectron spectroscopy measurement (Supplementary Fig. 14), and the conduction band was estimated using the band gap derived from the absorption band edge (Fig. 1b). A cross-sectional image of the device shows the formation of the discrete $\mathrm{CsPbI}_{3}$ particles layer with a thickness of $\sim 40 \mathrm{~nm}$ (Fig. 4b). We note that the residual IZI can locate between the discrete particles, which can prevent the device from short-circuit current and enhance the light-outcoupling efficiency, and similar studies have been demonstrated early ${ }^{37-39}$. The current density and luminance versus voltage $(J-L-V)$ and EQE curves of the device with different contents of IZI are shown in Supplementary Fig. 15. The electroluminescence (EL) peak is located at $\sim 698 \mathrm{~nm}$ and the shape remains unchanged under different bias voltages (Fig. 4c). The champion device based on the IZI-CsPbI ${ }_{3}$ film (mole ratio of 4:1.5:1 for IZI:CsI:PbI ${ }_{2}$ ) exhibits a peak EQE of $10.4 \%$ with luminance of $340 \mathrm{~cd} \mathrm{~m}^{-2}$, and the turn-on voltage is as low as $2 \mathrm{~V}$ (Fig. $4 \mathrm{~d}$, e). We note that our device peak EQE is a record for $3 \mathrm{D} \mathrm{CsPbI}_{3}$ film-based red $\mathrm{LEDs}^{40,41}$. Moreover, the efficiency roll-off of the device is significantly suppressed, and the EQE remains high ( $8 \%)$, under a high current density of $100 \mathrm{~mA} \mathrm{~cm}^{-2}$ (Fig. 4e). This feature is very different compared with previous $\mathrm{QD}$-based $\mathrm{CsPbI}_{3} \mathrm{LED}$ devices $^{21,26}$, where high EQE can only be obtained at low excitations likely due to the strong nonradiative Auger process. In addition, the IZI-CsPbI ${ }_{3}$ devices exhibit highly reproducible with average EQE of $8.1 \%$ for 75 devices (Supplementary Fig. 15c). The best device shows a half-lifetime of $20 \mathrm{~min}$ at a constant current density of $100 \mathrm{~mA} \mathrm{~cm}^{-2}$, and the EL peak position remains constant over time (Fig. 4f).

\section{Discussion}

In summary, we have developed a low-temperature method of forming $\mathrm{CsPbI}_{3}$ black phases for high performance $\mathrm{CsPbI}_{3} \mathrm{LED}$ applications via synergistic effect of IZI and $\mathrm{ZnO}$ electron transport layer. The judicious amount of $\mathrm{IZ}^{+}$in the precursor promotes the intermediate phase formation, followed by the formation of $\gamma$ $\mathrm{CsPbI}_{3}$ through the interionic exchange of $\mathrm{IZ}^{+}$with external Cs ${ }^{+}$in the process of the deprotonation of $\mathrm{IZ}^{+}$with $\mathrm{ZnO}$. The phase transition engineering can efficiently reduce the formation energy of $\mathrm{CsPbI}_{3}$ black phase, and facilitate the formation of discrete $\mathrm{CsPbI}_{3}$ particles film with high PLQE and long-term stability. The resulting $\mathrm{CsPbI}_{3}$ LED shows a peak EQE of $10.4 \%$ with suppressed efficiency roll-off. Importantly, the low-temperature formation process can be generally observed with various $\mathrm{RNH}_{3}{ }^{+}$-based large organic cations. So we believe that our work provides useful perspectives to tune the phase transition pathway, and offers an effective approach to fabricate low-temperature processed $\mathrm{CsPbI}_{3}$ black phase film for LEDs applications.

\section{Methods \\ Synthesis of $\mathrm{ZnO}$ colloidal solution. $\mathrm{ZnO}$ were synthesized by following the previously reported method ${ }^{27}$. The dimethyl sulfoxide solution of $\mathrm{Zn}(\mathrm{Ac})_{2} \cdot 2 \mathrm{H}_{2} \mathrm{O}$ $(3 \mathrm{mmol}$ in $30 \mathrm{~mL}$ ) was mixed in ethanol solution of tetramethylammonium hydroxide pentahydrate (TMAH. $\left.5 \mathrm{H}_{2} \mathrm{O}\right)(5.6 \mathrm{mmol}$ in $10 \mathrm{~mL})$ and stirred at $30^{\circ} \mathrm{C}$ for $24 \mathrm{~h}$. The $\mathrm{ZnO}$ colloids were precipitated with ethyl acetate, and washed it three times with ethanol and ethyl acetate. Finally, the obtained $\mathrm{ZnO}$ colloid were dis- persed in ethanol and set aside in the fridge until serve.}

Synthesis of organic ammonium salt. IZI was prepared by mixing IZ (2 g) and excess hydroiodic acid ( $45 \mathrm{wt} \%$ in water) in $15 \mathrm{~mL}$ of ethanol at $0{ }^{\circ} \mathrm{C}$. After the reaction mixture was stirred for $2 \mathrm{~h}, 60 \mathrm{~mL}$ diethyl ether was added into the mixture to obtain the precipitates. The collected precipitates were washed three times with diethyl ether and stored in an oven. BAI, HAI, PEAI, and NMAI were prepared by similar method.

Perovskite precursor solutions preparation. The $\mathrm{CsPb}_{3}$ precursor solution were prepared by dissolving IZI, CsI, and $\mathrm{PbI}_{2}$ with a molar ratio of $x$ in DMF at weight percent (wt\%) of $6 \%$ (IZI and $\mathrm{PbI}_{2}$ molar ratio is $x: 1, x=0,1,2,3,4$, CsI and $\mathrm{PbI}_{2}$ molar ratio fixed at 1.5:1), and the solution with molar ratio of $x=4$ is referred as IZI-CsPbI ${ }_{3}$. The IZI-PbI 2 precursor solutions were prepared by dissolving IZI and $\mathrm{PbI}_{2}$ with a molar ratio of 4:1 in DMF. BAI-CsPbI $\mathrm{H}_{3}, \mathrm{HAI}-\mathrm{CsPb}_{3}, \mathrm{PEAI}-\mathrm{CsPbI}_{3}$, and NMAI-CsPbI ${ }_{3}$ precursor solution were prepared by dissolving BAI, HAI, PEAI, NMAI in DMF solution of CsI and $\mathrm{PbI}_{2}$ with molar ratio of 4:1.5:1, 2:1.5:1, 4:1.5:1, 2:1.5:1, respectively.

Perovskite film deposition. $\mathrm{ZnO}$ colloidal solution was deposited onto the ITO substrate using spin-coating technique at $4000 \mathrm{rpm}$ for $45 \mathrm{~s}$, followed by annealing at $150^{\circ} \mathrm{C}$ for $30 \mathrm{~min}$ The PEIE $\left(1.5 \mathrm{mg} \mathrm{mL}^{-1}\right.$ in methoxyethanol) was spin coated onto the $\mathrm{ZnO}$ films at a speed of $5000 \mathrm{rpm}$ and annealed at $100^{\circ} \mathrm{C}$ for $10 \mathrm{~min}$. Finally, the precursor solution was spin coated onto ITO/ZnO/PEIE substrate (4000 rpm, $30 \mathrm{~s}$ ) or ITO substrate with $100^{\circ} \mathrm{C}$ annealing for various time to form films, respectively.

Device fabrication. The devices were fabricated with a structure of ITO/ZnO/ $\mathrm{PEIE} /$ perovskite/TFB/MoOx/Au. After the deposition of the $\mathrm{ZnO}$, PEIE and perovskite films as mentioned above, the TFB $\left(12 \mathrm{mg} \mathrm{mL}^{-1}\right.$ in chlorobenzene) layer was spin coated by $3000 \mathrm{rpm}$ for $30 \mathrm{~s}$. Finally, $\mathrm{MoO}_{x}(7 \mathrm{~nm})$ and $\mathrm{Au}(80 \mathrm{~nm})$ were deposited by thermal evaporation, respectively

Perovskite film characterization. XRD measurements were performed with a Rigaku Smart lab (3 kW) XRD patterns with Bragg-Brentano focusing, a diffracted beam monochromator and a conventional $\mathrm{Cu}$ target X-ray tube set to $40 \mathrm{kV}$ and $30 \mathrm{~mA}$. Time-dependent UV-vis absorption spectra were obtained on PerkinElmer Lambda 950 spectrometer. The general morphologies of the films were characterized by FEI (Quanta 200 FEG) SEM under a voltage of $5 \mathrm{kV}$. XPS tests were carried out using a Thermo ESCALAB250 Xi X-ray photoelectron spectrometer with $\mathrm{Al} \mathrm{K} \mathrm{K}_{\alpha} \mathrm{X}$-ray as the excitation source. All binding energies were referred to the $\mathrm{C} 1 s$ peak at $284.8 \mathrm{eV}$ of the surface areas of the samples. ATR-FTIR spectra of films were characterized by a Thermo-Niclet IS50 equipped with a Smart SAGA reflectance accessory in the range of $450-4000 \mathrm{~cm}^{-1}$. PL spectra were obtained using a fluorescent spectrophotometer (F-4600, HITACHI) with a 200 W Xe lamp as an excitation source. The Excitation-intensity-dependent PLQE of perovskite films was monitored by a joint control of a $450 \mathrm{~nm}$ continuous wave laser, $1000 \mu \mathrm{m}$ slit width, optical fiber spectrometer, and integrating sphere ${ }^{42}$. The film thickness was determined by a surface profiler (KLA-Tencor).

Kinetic modeling of the phase transition. The dependence of rate on temperature indicates a significant activation energy barrier $\left(E_{\mathrm{a}}\right)$ for the process of the $\gamma$ $\mathrm{CsPbI}_{3}$ formation. We worked out the activation energy barrier using the Mittemeijer model: ${ }^{36}$

$$
\ln \left(t_{\mathrm{x} 2}-t_{\mathrm{x} 1}\right)=\frac{E_{\mathrm{a}}}{R T}-\ln k_{0}+\ln \left(\beta_{\mathrm{x} 2}-\beta_{\mathrm{x} 1}\right),
$$

where $E_{\mathrm{a}}$ is the effective activation energy barrier, $t_{\mathrm{x} 1}$ and $t_{\mathrm{x} 2}$ are the annealing time at which the transformed fraction is $\chi(t)=0.2$ and $0.8, R$ is the gas constant, $T$ is the temperature, and $k_{0}$ is a rate constant prefactor.

Device characterization. The LED was measured in glove box at room temperature, and detailed setup can be found in reference ${ }^{38}$. A Keithley 2400 source meter with a step of $0.05 \mathrm{~V} \mathrm{~s}^{-1}$ and a fiber integration sphere (FOIS-1) coupled with a QE65 Prospectrometer were used for the device measurements. The device area is $7.25 \mathrm{~mm}^{2}$. The device lifetime was measured by using the same setup under a constant current density of $100 \mathrm{~mA} \mathrm{~cm}^{-2}$.

\section{Data availability}

The data that support the finding of this study are available from the corresponding author upon reasonable request.

Received: 2 March 2020; Accepted: 18 June 2020;

Published online: 21 September 2020

\section{References}

1. Yantara, N. et al. Inorganic halide perovskites for efficient light-emitting diodes. J. Phys. Chem. Lett. 6, 4360-4364 (2015).

2. Zhang, S. et al. Efficient red perovskite light-emitting diodes based on solution-processed multiple quantum wells. Adv. Mater. 29, 1606600 (2017). 
3. Jeong, B. et al. All-inorganic $\mathrm{CsPbI}_{3}$ perovskite phase-stabilized by poly (ethylene oxide) for red-light-emitting diodes. Adv. Funct. Mater. 28, 1706401 (2018).

4. Wang, Y. et al. Thermodynamically stabilized $\beta-\mathrm{CsPb}_{3}$-based perovskite solar cells with efficiencies $>18 \%$. Science 365, 591-595 (2019).

5. Wang, K. et al. All-inorganic cesium lead iodide perovskite solar cells with stabilized efficiency beyond 15\%. Nat. Commun. 9, 4544 (2018).

6. Tsui, K. Y., Onishi, N. \& Berger, R. F. Tolerance factors revisited geometrically designing the ideal environment for perovskite dopants. J. Phys. Chem. C 120, 23293-23298 (2016).

7. Sutton, R. J. et al. Cubic or orthorhombic? revealing the crystal structure of metastable black-phase $\mathrm{CsPbI}_{3}$ by theory and experiment. ACS Energy Lett. 3, 1787-1794 (2018).

8. Marronnier, A. et al. Anharmonicity and disorder in the black phases of cesium lead iodide used for stable inorganic perovskite solar cells. ACS Nano 12, 3477-3486 (2018).

9. Stoumpos, C. C., Malliakas, C. D. \& Kanatzidis, M. G. Semiconducting tin and lead iodide perovskites with organic cations: phase transitions, high mobilities, and near-infrared photoluminescent properties. Inorg. Chem. 52, 9019-9038 (2013).

10. Stoumpos, C. C. \& Kanatzidis, M. G. The renaissance of halide perovskites and their evolution as emerging semiconductors. Acc. Chem. Res. 48, 2791-2802 (2015).

11. Steele, J. A. et al. Thermal unequilibrium of strained black $\mathrm{CsPbI}_{3}$ thin films. Science 365, 679-684 (2019).

12. Sun, J. K. et al. Polar solvent induced lattice distortion of cubic $\mathrm{CsPbI}_{3}$ nanocubes and hierarchical self-assembly into orthorhombic single-crystalline nanowires. J. Am. Chem. Soc. 140, 11705-11715 (2018)

13. Eperon, G. E. et al. Inorganic caesium lead iodide perovskite solar cells. $J$. Mater. Chem. A 3, 19688-19695 (2015).

14. Wang, Y., Zhang, T., Kan, M. \& Zhao, Y. Bifunctional stabilization of allinorganic $a-\mathrm{CsPbI}_{3}$ perovskite for $17 \%$ efficiency photovoltaics. J. Am. Chem. Soc. 140, 12345-12348 (2018).

15. Noel, N. K. et al. Unveiling the influence of $\mathrm{pH}$ on the crystallization of hybrid perovskites, delivering low voltage loss photovoltaics. Joule 2, 328-343 (2017).

16. Wang, $\mathrm{Y}$. et al. The role of dimethylammonium iodide in $\mathrm{CsPb}_{3}$ perovskite fabrication: additive or dopant? Angew. Chem. Int. Ed. 58, 16691-16696 (2019).

17. Wu, M., Yan, K., Wang, Y. \& Kang, X. High crystallinity and photovoltaic performance of $\mathrm{CsPb}_{3}$ film enabled by secondary dimension. J. Energy Chem. 48, 181-186 (2020).

18. Ke, W., Spanopoulos, I., Stoumpos, C. C. \& Kanatzidis, M. G. Myths and reality of $\mathrm{HPbI}_{3}$ in halide perovskite solar cells. Nat. Commun. 9, 4785 (2018).

19. Pei, Y. et al. Unveiling property of hydrolysis-derived $\mathrm{DMAPbI}_{3}$ for perovskite devices: composition engineering, defect mitigation, and stability optimization. iScience 15, 165-172 (2019).

20. Hutter, E. M. et al. Vapour-deposited cesium lead iodide perovskites: microsecond charge carrier lifetimes and enhanced photovoltaic performance. ACS Energy Lett. 2, 1901-1908 (2017).

21. Chiba, T. et al. Anion-exchange red perovskite quantum dots with ammonium iodine salts for highly efficient light-emitting devices. Nat. Photonics 12, 681-687 (2018).

22. Shen, $\mathrm{X}$. et al. $\mathrm{Zn}$-alloyed $\mathrm{CsPbI}_{3}$ nanocrystals for highly efficient perovskite light-emitting devices. Nano Lett. 19, 1552-1559 (2019).

23. $\mathrm{Li}, \mathrm{G}$. et al. Surface ligand engineering for near-unity quantum yield inorganic halide perovskite QDs and high-performance QLEDs. Chem. Mater. 30, 6099-6107 (2018).

24. Lu, M. et al. Simultaneous strontium doping and chlorine surface passivation improve luminescence intensity and stability of $\mathrm{CsPbI}_{3}$ nanocrystals enabling efficient light-emitting devices. Adv. Mater. 30, 1804691 (2018).

25. Pan, J. et al. Bidentate ligand-passivated $\mathrm{CsPbI}_{3}$ perovskite nanocrystals for stable near-unity photoluminescence quantum yield and efficient red lightemitting diodes. J. Am. Chem. Soc. 140, 562-565 (2017).

26. Lu, M. et al. Spontaneous silver doping and surface passivation of $\mathrm{CsPb}_{3}$ perovskite active layer enable light-emitting devices with an external quantum efficiency of 11.2\%. ACS Energy Lett. 3, 1571-1577 (2018).

27. Wang, J. et al. Interfacial control toward efficient and low-voltage perovskite light-emitting diodes. Adv. Mater. 27, 2311-2316 (2015).

28. Bhargava, G., Ramanarayanan, T. A. \& Bernasek, S. L. Imidazole-Fe interaction in an aqueous chloride medium: effect of cathodic reduction of the native oxide. Langmuir 26, 215-219 (2010).

29. Liu, W. et al. Simultaneous catalyzing and reinforcing effects of imidazolefunctionalized graphene in anhydride-cured epoxies. J. Mater. Chem. 22, 18395-18402 (2012).

30. Winiarski, J., Tylus, W., Winiarska, K., Szczygieł, I. \& Szczygieł, B. XPS and FT-IR characterization of selected synthetic corrosion products of zinc expected in neutral environment containing chloride ions. J. Spectrosc. 2018, 1-14 (2018).
31. Nicholas, N. J., Franks, G. V. \& Ducker, W. A. The mechanism for hydrothermal growth of zinc oxide. CrystEngComm 14, 1232-1240 (2012).

32. Wang, M., Jiang, L., Kim, E. J. \& Hahn, S. H. Electronic structure and optical properties of $\mathrm{Zn}(\mathrm{OH})_{2}$ : LDA $+U$ calculations and intense yellow luminescence. RSC Adv. 5, 87496-87503 (2015).

33. Lefatshe, K., Muiva, C. M. \& Kebaabetswe, L. P. Extraction of nanocellulose and in-situ casting of $\mathrm{ZnO} /$ cellulose nanocomposite with enhanced photocatalytic and antibacterial activity. Carbohydr. Polym. 164, 301-308 (2017).

34. Xue, G., Dai, Q. \& Jiang, S. Chemical reactions of imidazole with metallic silver studied by the use of SERS and XPS techniques. J. Am. Chem. Soc. 110 2393-2395 (1988).

35. Yuan, Z. et al. Unveiling the synergistic effect of precursor stoichiometry and interfacial reactions for perovskite light-emitting diodes. Nat. Commun. 10, 2818 (2019).

36. Moore, D. T. et al. Crystallization kinetics of organic-inorganic trihalide perovskites and the role of the lead anion in crystal growth. J. Am. Chem. Soc 137, 2350-2358 (2015).

37. Zhao, X. \& Tan, Z.-K. Large-area near-infrared perovskite light-emitting diodes. Nat. Photonics 14, 215-218 (2020)

38. Cao, Y. et al. Perovskite light-emitting diodes based on spontaneously formed submicrometre-scale structures. Nature 562, 249-253 (2018)

39. Wang, $\mathrm{H}$. et al. Perovskite-molecule composite thin films for efficient and stable light-emitting diodes. Nat. Commun. 11, 891 (2020).

40. Ke, Y. et al. Defect passivation for red perovskite light-emitting diodes with improved brightness and stability. J. Phys. Chem. Lett. 10, 380-385 (2019).

41. Han, B. et al. Stable, efficient red perovskite light-emitting diodes by $(\alpha, \delta)$ $\mathrm{CsPbI}_{3}$ phase engineering. Adv. Funct. Mater. 28, 1804285 (2018).

42. de Mello, J. C., Wittmann, H. F. \& Friend, R. H. An improved experimental determination of external photoluminescence quantum efficiency. Adv. Mater. 9, 230-232 (1997)

\section{Acknowledgements}

This work is financially supported by the Major Research Plan of the National Natura Science Foundation of China (91733302), the National Natural Science Foundation of China $(51703094,61935017,61974066)$, the Natural Science Foundation of Jiangsu Province, China (BK20170991), the National Science Fund for Distinguished Young Scholars (61725502), Major Program of Natural Science Research of Jiangsu Higher Education Institutions of China (18KJA510002), the National Key Research and Development Program of China (2018YFB0406704), and the Natural Science Fund for Colleges and Universities in Jiangsu Province of China (17KJB150023). The work at Linköping is funded by the ERC Starting Grant (717026) and the Swedish Government Strategic Research Area in Materials Science on Functional Materials at Linköping University (Faculty Grant SFO-Mat-LiU no. 2009-00971). X.-K.L. is a Marie SkłodowskaCurie Fellow (No. 798861). F.G. is a Wallenberg Academy Fellow. We thank Dr. Xianjie Liu for XPS measurement and analysis. We thank Dr. Zhangjun Hu for FTIR measurement and analysis. Open Access funding provided by Linköping University.

\section{Author contributions}

J.W. and C.Y. had the idea for and designed the experiments. J.W., W.H., and F.G. supervised the work. C.Y. and C.L. carried out the device fabrication and characterizations and films characterizations. K.W. and B.M. carried out the PLQE characterizations The $\mathrm{ZnO}$ was synthesized with the assistance of C.K. and N.F. The single crystal of $\mathrm{IZPbI}_{3}$ was cultivated with the assistance of F.J. and C.X. C.T., Y.Z., and H.Z. conducted the SEM measurements. Y.Y. conducted the XPS measurement. C.Y. wrote the paper. J.W., F.G., W.H., X.-K.L., and R.L. provided revisions. All authors discussed the results and commented on the paper.

\section{Competing interests}

The authors declare no competing interests.

\section{Additional information}

Supplementary information is available for this paper at https://doi.org/10.1038/s41467 020-18380-1.

Correspondence and requests for materials should be addressed to F.G., W.H. or J.W.

Peer review information Nature Communications thanks Takayuki Chiba, Zhi-Kuang Tan and the other, anonymous, reviewer(s) for their contribution to the peer review of this work. Peer reviewer reports are available.

Reprints and permission information is available at http://www.nature.com/reprints

Publisher's note Springer Nature remains neutral with regard to jurisdictional claims in published maps and institutional affiliations. 
(c) (i) Open Access This article is licensed under a Creative Commons Attribution 4.0 International License, which permits use, sharing, adaptation, distribution and reproduction in any medium or format, as long as you give appropriate credit to the original author(s) and the source, provide a link to the Creative Commons license, and indicate if changes were made. The images or other third party material in this article are included in the article's Creative Commons license, unless indicated otherwise in a credit line to the material. If material is not included in the article's Creative Commons license and your intended use is not permitted by statutory regulation or exceeds the permitted use, you will need to obtain permission directly from the copyright holder. To view a copy of this license, visit http://creativecommons.org/ licenses/by/4.0/.

(C) The Author(s) 2020 